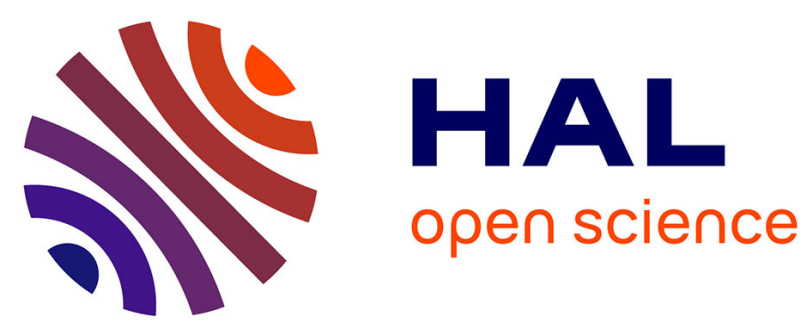

\title{
Performance evaluation of intra-site coordination schemes in cellular networks
}

\author{
Ahlem Khlass, Thomas Bonald, Salah Eddine Elayoubi
}

\section{To cite this version:}

Ahlem Khlass, Thomas Bonald, Salah Eddine Elayoubi. Performance evaluation of intra-site coordination schemes in cellular networks. Performance Evaluation, 2016, 10.1016/j.peva.2015.12.001 . hal-01280280

\section{HAL Id: hal-01280280 \\ https://hal.science/hal-01280280}

Submitted on 29 Feb 2016

HAL is a multi-disciplinary open access archive for the deposit and dissemination of scientific research documents, whether they are published or not. The documents may come from teaching and research institutions in France or abroad, or from public or private research centers.
L'archive ouverte pluridisciplinaire HAL, est destinée au dépôt et à la diffusion de documents scientifiques de niveau recherche, publiés ou non, émanant des établissements d'enseignement et de recherche français ou étrangers, des laboratoires publics ou privés. 


\title{
Performance Evaluation of Intra-Site Coordination Schemes in Cellular Networks
}

\author{
Ahlem Khlass ${ }^{\mathrm{a}, \mathrm{b}, *}$, Thomas Bonald ${ }^{\mathrm{a}}$, Salah Eddine Elayoubi ${ }^{\mathrm{b}}$ \\ ${ }^{a}$ Telecom ParisTech, 46 Rue Barrault, 75013 Paris, France \\ ${ }^{b}$ Orange Labs, 38-40, rue du General Leclerc, 92794 Issy-les-moulineaux, France
}

\begin{abstract}
In this work, we develop performance evaluation models for some intrasite coordination schemes in cellular networks, with a focus on Multi-flow transmission in HSPA+. We first focus on a static scheme where coordination is always performed for all users in the overlapping region of two cells. Through the analysis of a flow-level model, we show that this scheme indeed improves the cell-edge throughput at low loads but may make the system unstable at high loads, due to the suboptimal allocation of radio resources. We notice also that these results are very sensitive to the coordination threshold. We thus investigate a dynamic scheme which performs coordination according to resource availability in the site and show that this scheme outperforms the static one at all traffic loads independently of the coordination threshold. Additional results demonstrate an efficient load balancing ability across cells. We finally analyze how our models extend to the case of opportunistic scheduling schemes, like Proportional Fair, and show the impact of these schemes on the coordination gains.
\end{abstract}

Keywords: Cellular networks, HSPA+, multi-point transmission, sector coordination, flow-level modeling, queuing theory.

\footnotetext{
${ }^{*}$ Corresponding author.

Email addresses: ahlem.khlass@telecom-paristech.fr (Ahlem Khlass), thomas.bonald@telecom-paristech.fr (Thomas Bonald), salaheddine.elayoubi@orange.com (Salah Eddine Elayoubi)
} 


\section{Introduction}

Inter-cell interference is a major issue in cellular networks, especially with frequency reuse one. It does not only make users at the cell edge suffer from low throughputs but also decreases the overall network capacity since these users consume a significant proportion of the radio resources [1]. Cell coordination has been proposed as an efficient way for reducing inter-cell interference by either silencing some base stations or allowing several base stations to transmit data simultaneously to the same user [2].

Recently, it has been adopted by 3GPP standards for Beyond 3G networks. Release 11 introduced cell coordination for High Speed Packet Access $(\mathrm{HSPA}+)$ systems in the Multipoint transmission feature including three main techniques: the single point data transmission, the multi flow data transmission and the single frequency network data transmission [3]. In LTE-Advanced, cell coordination is included in Release 11 under the name of Coordinated MultiPoint (CoMP) [4]. Two main categories are proposed: the joint processing scheme and the joint scheduling/ beamforming scheme.

There are two broad classes of cell coordination schemes: inter-site coordination, where cells of different sites are coordinated, and intra-site coordination, where cells (or sectors) of the same site are coordinated [5][6]. Inter-site coordination remains critical to implement for several reasons related mainly to backhaul limitations. Thus, we focus on intra-site coordination which is much simpler and more practical since all scheduling decisions are local so there is no additional signaling overhead to be considered.

The major challenge of cell coordination is to control the strong coupling of scheduling decisions in the different cells. Indeed, scheduling a user in a given cell may prevent users in neighboring cells to be served, leading to the following dilemma:

1. Cell coordination increases substantially the data rates of users when scheduled.

2. Cell coordination leads to situations where only few users are simultaneously scheduled in the network, thus decreasing the proportion of time where individual users are scheduled.

This tradeoff between higher achievable rates (due to lower interference) and higher radio resource consumption (due to silences or joint transmissions to the same user) is arguably the key issue when designing a coordination scheme. 
The present work aims at evaluating the performance of intra-site coordination in cellular networks by addressing this tradeoff analytically, and numerically in a HSPA+ system. We start by investigating a static scheme where coordination is always performed in the overlapping area between two cells. We show in particular that this scheme improves cell-edge throughput at low loads but may make the system unstable at high loads, due to the suboptimal allocation of radio resources. We notice also that these results are very sensitive to the coordination area size. Hence, we investigate a dynamic scheme which performs coordination according to the resource availability in the site in the different sectors and we show that it outperforms the static one in all load situations, independently of the coordination area. Additional results demonstrate an efficient load balancing ability across cells. Finally, we investigate the impact of opportunistic scheduling algorithms on the coordination gains.

In summary, the main contributions of this paper are threefold:

- We develop an analytical framework for evaluating the performance of intra-site coordination in terms of throughput and load balancing.

- We analyze two different coordination schemes and their ability to improve cell edge throughput while preserving the system stability at high loads.

- We provide numerical performance results for a HSPA+ system.

The remainder of this paper is organized as follows. We start by presenting the related work. Then, we describe the cell coordination schemes. We present the model and we analyze performance in the absence of coordination in sections 4 and 5. Sections 6 and 7 are dedicated to the performance analysis of the static and dynamic schemes, respectively. In section 8, we investigate the impact of opportunistic scheduling algorithms on the coordination gains. Section 9 concludes the paper.

\section{Related work}

The idea of cell coordination was originally used in cell breathing methods applied to multiple cellular networks [7] which allows overloaded cells to offload traffic to neighboring cells and more explicitly in the soft handover

mechanism in WCDMA system where a user is simultaneously associated to 
two or more base stations in order to enhance the system performance in terms of QoS and coverage [8].

Recently, within the framework of Release 11 of HSPA + [3], the concept of Multi-point transmission has been introduced. It is expected to enhance user experience by improving network performance, especially at the cell edge, and efficiently balancing load between cells as it has been shown in several studies, see e.g. [9, 10, 11] and references therein. Equivalently, Release 11 of LTE-Advanced system has adopted the Coordinated MultiPoint transmission as a key feature among with other advanced technologies such as Multi-User MIMO (see [5] and [12] for instance).

The first attempts to evaluate the performance of coordination schemes (see e.g. [2] and references therein) focused on physical layer aspects and were based on so-called full buffer simulations, as proposed by 3GPP [13]. In this approach, a fixed number of users is simulated in each cell with a nearly complete emulation of the physical and MAC layers. The advantage of these simulations is their high accuracy with respect to the lower layers as they take into account a complete channel model including path loss, shadowing and fast fading. However, they do not capture the dynamic aspect of traffic, which is critical for the performance of coordination schemes.

Due to this shortcoming, so-called finite buffer simulators have been recently proposed (see the FTP simulation model in [13]). These simulations add the dynamic traffic layer whereby short-lived data flows are generated at random times, mimicking the user behavior. Such simulations have recently been used in [14] to evaluate the performance of coordination schemes in HSPA networks and in [15] to analyze its ability to balance load. Substantial throughput gains from coordination are observed, especially at the cell edge. Although finite buffer simulations provide useful insights into the efficiency of coordination schemes, they are computationally intensive, especially when stability issues are addressed.

Analytical methods are practically interesting and, to the best of our knowledge, have not yet been proposed in the considered dynamic setting with flow arrivals and departures. Indeed, the underlying model corresponds to a set of coupled queues, which is known to be intractable beyond some very specific cases [16]. Bounds and approximations for the impact of intercell interference on user throughput have been derived in [17], [18], [19], [20], and [21].In [22], flow level Markovian analysis was used to evaluate the performance of coordination using LTE-Advanced codebook-based schemes jointly with Multi user diversity. Such studies are very relevant but are not 


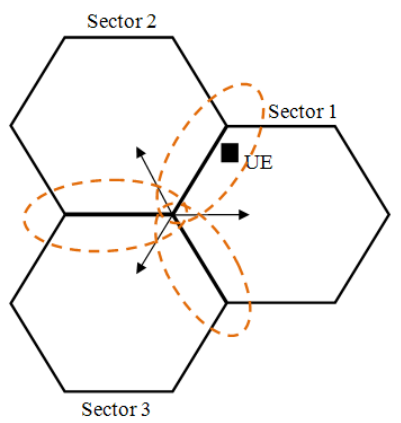

Figure 1: A tri-sector site with the coordination regions between sectors.

scalable.

Note that preliminary results on intra-site coordination for HSPA + systems have been presented in our conference paper [23]. The present work extends [23] by studying the sensitivity of the schemes to the coordination area size, exploring further the cell coordination ability to balance load efficiently between non-uniformly loaded sectors and investigating the impact of physical layer aspects (fast fading, opportunistic scheduling) on coordination gains.

\section{Cell coordination schemes}

We describe in the following the two different intra-site coordination schemes that are analyzed in this work.

\subsection{Static scheme}

In the absence of coordination, each user equipment (UE) is associated and scheduled by the so-called serving sector which offers the best signal quality. However, when multi-point (MP) transmission is enabled, each UE in the overlapping area between two sectors (typically the handover region [3]) can be scheduled by both. This coordination region is determined by some threshold, say $\delta$, on the ratio between the pilot channels of the serving and the adjacent sectors. This is illustrated for a tri-sector site in figure 1. We focus on one of the possible multi-point transmission techniques where a user in the coordination region receives simultaneously different transport blocks from two different sectors. This corresponds to the so-called multi-flow (MF) transmission feature in HSPA+ systems and to Joint Processing/transmission 
(JP/JT) in LTE-Advanced systems. We refer to this scheme as static since a UE is simultaneously scheduled by two sectors whenever it lies in the coordination region of these sectors, independently of the network state. We will see that this scheme is only beneficial at low loads; at high loads, it brings an additional load which maintains the network in congestion due to the lack of available resources. Indeed, the performance of the static scheme is too sensitive to the coordination threshold and thereby to the size of the coordination area.

\subsection{Dynamic scheme}

We also investigate a dynamic scheme which performs coordination according to resource availability in the different sectors. In fact, a UE in the coordination region receives data from the non-serving sector only if the latter has no UE to serve in its serving zone. We refer to this scheme as dynamic since the coordination decisions depend on the instantaneous state of the sectors and vary rapidly with time. In [24], a dynamic scheme is described where the coordination decisions are based on the channel Quality Information (CQI) reported to the NodeB. However, in this work, we only consider the load information.

Note that the condition imposed on the coordination decisions may look too restrictive at first sight, since only empty sectors coordinate with other sectors. Our results of section 7 indicate that this is not the case. The reason is that, even at medium loads, the probability that a sector is empty is significant. At high loads, coordination is almost always deactivated which preserves the network stability. Hence, the system performance is no more sensitive to the coordination threshold.

\section{Model}

\subsection{SINR analysis}

We consider a radio site consisting of $K$ sectors indexed by $k$. The signalto-noise-plus-interference ratio (SINR) is analyzed as follows.

- Classical transmission

In the absence of coordination, the SINR of UE $u$ served by sector $s$ is given by:

$$
\mathrm{SINR}_{u, s}=\frac{P_{u, s}}{\sum_{k \neq s} P_{u, k}+I_{u}+N},
$$


where $P_{u, k}$ denotes the power received by UE $u$ from sector $k$ of the same site, $I_{u}$ is the received inter-site interference and $N$ is the thermal noise. The resulting data rate is $f\left(\mathrm{SINR}_{u, s}\right)$ for some function $f$; a standard model for $f$ is the Shannon capacity of the Gaussian channel $f(s)=W \log (1+s)$, where $W$ is the channel bandwidth. This only provides an upper bound, assuming Gaussian noise and interference; more realistic values derived from real systems are used in $§ 6.4 .1$.

- Multi-point transmission

When coordination is performed between two adjacent sectors, the UE $u$ receives simultaneously two different transport blocks from the serving sector $s$ and from the coordinating sector $c$, leading to two flows with respective SINRs:

$$
\operatorname{SINR}_{u, s}^{M P}=\frac{P_{u, s}}{\epsilon P_{u, c}+\sum_{k \neq s, c} P_{u, k}+I_{u}+N},
$$

and

$$
\operatorname{SINR}_{u, c}^{M P}=\frac{P_{u, c}}{\epsilon P_{u, s}+\sum_{k \neq s, c} P_{u, k}+I_{u}+N}
$$

where $\epsilon$ denotes the receiver capability of canceling the interference from the other sectors within a given site. The data rate is then the sum $f\left(\mathrm{SINR}_{u, s}^{M P}\right)+$ $f\left(\mathrm{SINR}_{u, c}^{M P}\right)$ which is higher than in the absence of coordination, as illustrated in $§ 6.4 .1$.

\subsection{Scheduling algorithm}

The coordination decisions are taken by a centralized scheduler at the site level. Although the scheduling algorithm should in practice exploit multiuser diversity in order to improve the spectral efficiency of the system, we consider first a simple, fair scheduling algorithm that does not exploit fast fading. The impact of opportunistic scheduling is considered in section 8 .

\subsection{Traffic aspects}

\subsubsection{Traffic characteristics}

We consider elastic traffic. Data flows arrive according to a Poisson process of intensity $\bar{\lambda}_{k}$ in each sector $k$ of the reference site. Each flow stays in the system as long as the corresponding data have not been completely transmitted to the UE. Flow sizes are assumed to be independent and exponentially 
distributed with mean $\sigma$ bits, although all our results are approximately insensitive to the flow size distribution beyond the mean, as explained below. The traffic intensity in sector $k$ is $\bar{\lambda}_{k} \sigma$ in bit/s.

The transmission rate of a UE depends on its location in the cell. We consider an arbitrary set $S$ of classes of UEs indexed by $i$ to reflect the different radio conditions (i.e., locations) in the considered site. All UEs of the same class have approximately the same location and thus the same serving BS and the same data rate when scheduled.

We denote by $S_{k}$ the set of classes whose serving sector is $k$; UEs of class $i$ have transmission rate $R_{i}$ when scheduled without coordination and transmission rate $R_{i}^{\prime}$ when scheduled with coordination. The corresponding flows are referred to as class- $i$ flows in the following. A flow arriving in sector $k$ is of class $i \in S_{k}$ with probability $p_{i}$, with:

$$
\sum_{i \in S_{k}} p_{i}=1
$$

Thus class- $i$ flows arrive according to a Poisson process with intensity $\lambda_{i} \equiv$ $\bar{\lambda}_{k} p_{i}$ in sector $k$, contributing to the traffic intensity $\lambda_{i} \sigma$ (in bit/s).

\subsubsection{Throughput metric}

Let $X_{i}(t)$ be the number of class- $i$ flows at time $t$. The vector $\vec{X}(t)=$ $\left(X_{i}\right)_{i \in S}$ is an irreducible Markov process [25] whose transition rates depend on the considered coordination scheme, as described in the following sections. Assuming that this Markov process is ergodic, the mean duration $\tau_{i}$ of class- $i$ flows follows from Little's law [26] :

$$
\tau_{i}=\frac{E\left(X_{i}\right)}{\lambda_{i}}
$$

We define the flow throughput (in bit/s) as the ratio of the mean flow size (in bits) to the mean flow duration (in s). For class $i$, we get:

$$
\gamma_{i}=\frac{\sigma}{\tau_{i}}=\frac{\lambda_{i} \sigma}{E\left(X_{i}\right)}
$$

This is the ratio of the traffic intensity of class $i$ (in bit/s) to the mean number of class- $i$ flows. This throughput metric reflects user experience, accounting both for the radio conditions, through class $i$, and for the random nature of traffic, through the stationary distribution of the Markov process $\vec{X}(t)$. 
The mean flow throughput in sector $k$ is given by:

$$
\bar{\gamma}_{k}=\frac{\sigma}{\bar{\tau}_{k}}
$$

where $\bar{\tau}_{k}$ is the mean flow duration in sector $k$ :

$$
\bar{\tau}_{k}=\sum_{i \in S_{k}} p_{i} \tau_{i}
$$

We obtain:

$$
\bar{\gamma}_{k}=\left(\sum_{i \in S_{k}} \frac{p_{i}}{\gamma_{i}}\right)^{-1} .
$$

This is the weighted harmonic mean of the per-class flow throughputs, with weights given by the per-class traffic intensities.

\section{Analysis without coordination}

We start with the analysis of the reference system without coordination. All UEs in each sector $k$ are assumed to be served in a fair manner based on Round-Robin (RR) scheduler.

\subsection{Processor-sharing queues}

Consider class- $i$ flows in sector $k$. Since the mean flow size is $\sigma$, the mean scheduling time required to complete a class- $i$ flow is $\sigma / R_{i}$. Since class- $i$ flows arrive at rate $\lambda_{i}$, the load they generate is:

$$
\rho_{i}=\frac{\lambda_{i} \sigma}{R_{i}}
$$

At the flow time-scale, slots are very short (in the range of ms) so that the fair scheduling algorithm corresponds to a processor-sharing service discipline per sector. Specifically, the number of flows in sector $k$ evolves like the number of customers in a multi-class processor-sharing queue or equivalently to a Whittle network [27] with load:

$$
\bar{\rho}_{k}=\sum_{i \in S_{k}} \rho_{i} .
$$


Note that, in view of (1) and (4), we have:

$$
\bar{\rho}_{k}=\frac{\bar{\lambda}_{k} \sigma}{\bar{R}_{k}}
$$

where $\bar{R}_{k}$ is the harmonic mean transmission rate over sector $k$ :

$$
\bar{R}_{k}=\left(\sum_{i \in S_{k}} \frac{p_{i}}{R_{i}}\right)^{-1} .
$$

Under the stability condition $\bar{\rho}_{1}<1, \ldots, \bar{\rho}_{K}<1$ (no sector is in overload), the stationary distribution of the number of flows of each class in the site is given by:

$$
\pi(\vec{x})=\prod_{k=1}^{K}\left(1-\bar{\rho}_{k}\right) \frac{\bar{x}_{k} !}{\prod_{i \in S_{k}} x_{i} !} \prod_{i \in S_{k}} \rho_{i}^{x_{i}},
$$

where $\bar{x}_{k}$ denotes the number of active flows in sector $k$. Note that, in the absence of coordination, the sectors are mutually independent and the probability that sector $k$ is active (in the sense that $\bar{x}_{k}>0$ ) is equal to $\bar{\rho}_{k}$.

\subsection{Flow throughputs}

For any class $i \in S_{k}$, the mean number of class- $i$ flows is given by:

$$
E\left(X_{i}\right)=\sum_{\vec{x}} x_{i} \pi(\vec{x})=\frac{\rho_{i}}{1-\bar{\rho}_{k}} .
$$

From (2) and (4), we deduce the class- $i$ flow throughput:

$$
\gamma_{i}=R_{i}\left(1-\bar{\rho}_{k}\right)
$$

Thus the flow throughput of any UE of class $i$ is maximum and equal to its transmission rate $R_{i}$ when $\bar{\rho}_{k}=0$ (no traffic in sector $k$ ) and decreases linearly with the sector load $\bar{\rho}_{k}$ (due to the sharing of the transmission resource with other UEs). In view of (3) and (6), the mean flow throughput of sector $k$ is given by:

$$
\bar{\gamma}_{k}=\bar{R}_{k}\left(1-\bar{\rho}_{k}\right)
$$

\section{Static coordination scheme}

In this section, we focus on the static scheme where the coordination region is determined by a fixed threshold $\delta$ as described in $\S 3.1$. 


\subsection{Scheduling algorithm}

Since we neglect fast fading at this stage, the scheduling algorithm reduces to the random, iterative selection of UEs until all sectors having UEs to serve are busy. This is equivalent to proportional fair scheduler [28] when radio channel variations are slow or too fast to be exploited.

Note that a UE in the coordination region can be scheduled only if both its serving sector $s$ and its coordinating sector $c$ are available at the scheduling decision time.

\subsection{Coordinating two sectors}

To gain insight into the behavior of the static coordination scheme, we start with the simple case of $K=2$ sectors. We refer to zone 12 as the coordination region of sectors 1 and 2 . We denote by $C_{12} \subset S$ the set of classes in zone 12; UEs of class $i \in C_{12}$ are served simultaneously by sectors 1 and 2 at rate $R_{i}^{\prime}$. The sets $C_{1}=S_{1} \backslash C_{12}$ and $C_{2}=S_{2} \backslash C_{12}$ define zones 1 and 2 of sectors 1 and 2, respectively, where UEs are not subject to coordination; UEs of class $i \in C_{j}$ are served by sector $j$ at rate $R_{i}$, for $j=1,2$.

Let $Z_{j}(t)$ be the total number of flows in zone $j$ at time $t$, for $j=1,2,12$. Denote by $\vec{Z}(t)$ the corresponding vector. Whenever $\vec{Z}(t)=\vec{z}$, the considered scheduler selects UEs in zones 1 and 2 (assuming $z_{1}, z_{2}>0$ ) a fraction of time:

$$
\phi_{1}(\vec{z})=\phi_{2}(\vec{z})=\frac{z_{1}+z_{2}}{z_{1}+z_{2}+z_{12}},
$$

and UEs in zone 12 a fraction of time:

$$
\phi_{12}(\vec{z})=\frac{z_{12}}{z_{1}+z_{2}+z_{12}} .
$$

Indeed, since the coordination requires the simultaneous transmission from both sectors, the scheduling algorithm does not select a UE in zone 12 whenever the first randomly selected UE is either in zone 1 or in zone 2 . Note that this system corresponds to a two-link linear bandwidth-sharing network which has been investigated in [29] and references therein.

Since the scheduler is fair within each zone, the system corresponds to a set of three coupled processor-sharing queues with state-dependent service rates given by (10)-(11) and respective loads:

$$
\bar{\rho}_{1}=\frac{\bar{\lambda}_{1} \sigma}{\bar{R}_{1}}, \quad \bar{\rho}_{2}=\frac{\bar{\lambda}_{2} \sigma}{\bar{R}_{2}}, \quad \bar{\rho}_{12}=\frac{\bar{\lambda}_{12} \sigma}{\bar{R}_{12}^{\prime}},
$$


with arrival rates:

$$
\bar{\lambda}_{1}=\sum_{i \in C_{1}} \lambda_{i}, \quad \bar{\lambda}_{2}=\sum_{i \in C_{2}} \lambda_{i}, \quad \bar{\lambda}_{12}=\sum_{i \in C_{12}} \lambda_{i}
$$

and harmonic mean transmission rates:

$$
\bar{R}_{1}=\frac{\sum_{i \in C_{1}} p_{i}}{\sum_{i \in C_{1}} \frac{p_{i}}{R_{i}}}, \quad \bar{R}_{2}=\frac{\sum_{i \in C_{2}} p_{i}}{\sum_{i \in C_{2}} \frac{p_{i}}{R_{i}}},
$$

and

$$
\bar{R}_{12}^{\prime}=\frac{\sum_{i \in C_{12}} p_{i}}{\sum_{i \in C_{12}} \frac{p_{i}}{R_{i}^{\prime}}}
$$

Note that, unlike in zones 1 and 2, the transmission rates in zone 12 benefit from the coordination gains.

To be more specific, the queuing system is described by the following transition rates of the Markov process $\vec{Z}(t)$ from state $\vec{z}$ to state $\vec{z}$ :

$$
q\left(\vec{z}, \vec{z}^{\prime}\right)= \begin{cases}\bar{\lambda}_{j} & \text { for } \vec{z}^{\prime}=\vec{z}+\vec{e}_{j} j=1,2,12, \\ \phi_{j}(\vec{z}) \frac{\bar{R}_{j}}{\sigma_{\bar{R}_{12}^{\prime}}} & \text { for } \vec{z}^{\prime}=\vec{z}-\vec{e}_{j}, j=1,2 \\ \phi_{12}(\vec{z}) \frac{\text { for }}{\sigma} & \vec{z}^{\prime}=\vec{z}-\vec{e}_{12}\end{cases}
$$

where $\vec{e}_{j}$ is the unit vector on component $j$, for $j=1,2,12$. In view of (10)(11), this Markov process is reversible [30] (equivalently, the queuing system is a Whittle network). We deduce the stationary distribution of the Markov process $\vec{Z}(t)$ :

$$
\pi(\vec{z})=\frac{\left(1-\bar{\rho}_{1}-\bar{\rho}_{12}\right)\left(1-\bar{\rho}_{2}-\bar{\rho}_{12}\right)}{\left(1-\bar{\rho}_{12}\right)} \times\left(\begin{array}{c}
z_{1}+z_{2}+z_{12} \\
z_{1}+z_{2}
\end{array}\right) \bar{\rho}_{1}^{z_{1}} \bar{\rho}_{2}^{z_{2}} \bar{\rho}_{12}^{z_{12}},
$$

under the stability condition:

$$
\bar{\rho}_{1}+\bar{\rho}_{12}<1, \quad \bar{\rho}_{2}+\bar{\rho}_{12}<1 .
$$

Moreover, the results are insensitive to the flow size distribution beyond the mean [31]: it is not necessary to assume an exponential flow size distribution.

We deduce from the stationary distribution (14) the mean number of flows in each zone:

$$
E\left(Z_{1}\right)=\frac{\bar{\rho}_{1}}{1-\bar{\rho}_{1}-\bar{\rho}_{12}},
$$




$$
\begin{gathered}
E\left(Z_{2}\right)=\frac{\bar{\rho}_{2}}{1-\bar{\rho}_{2}-\bar{\rho}_{12}}, \\
E\left(Z_{12}\right)=\frac{\bar{\rho}_{12}}{1-\bar{\rho}_{12}}\left(1+\frac{\bar{\rho}_{1}}{1-\bar{\rho}_{1}-\bar{\rho}_{12}}+\frac{\bar{\rho}_{2}}{1-\bar{\rho}_{2}-\bar{\rho}_{12}}\right) .
\end{gathered}
$$

and from (2), the flow throughput in each zone:

$$
\begin{gathered}
\bar{\gamma}_{1}=\bar{R}_{1}\left(1-\bar{\rho}_{1}-\bar{\rho}_{12}\right), \quad \bar{\gamma}_{2}=\bar{R}_{2}\left(1-\bar{\rho}_{2}-\bar{\rho}_{12}\right), \\
\bar{\gamma}_{12}=\frac{\bar{R}_{12}^{\prime}\left(1-\bar{\rho}_{12}\right)}{1+\frac{\bar{\rho}_{1}}{1-\bar{\rho}_{1}-\bar{\rho}_{12}}+\frac{\bar{\rho}_{2}}{1-\bar{\rho}_{2}-\bar{\rho}_{12}}} .
\end{gathered}
$$

The mean number of flows of class $i$ being proportional to the load of this class in the corresponding queue, we also obtain the flow throughput of each class within each zone:

$$
\begin{gathered}
\forall i \in C_{1}, \quad \gamma_{i}=R_{i}\left(1-\bar{\rho}_{1}-\bar{\rho}_{12}\right), \\
\forall i \in C_{2}, \quad \gamma_{i}=R_{i}\left(1-\bar{\rho}_{2}-\bar{\rho}_{12}\right), \\
\forall i \in C_{12}, \quad \gamma_{i}=\frac{R_{i}^{\prime}\left(1-\bar{\rho}_{12}\right)}{1+\frac{\bar{\rho}_{1}}{1-\bar{\rho}_{1}-\bar{\rho}_{12}}+\frac{\bar{\rho}_{2}}{1-\bar{\rho}_{2}-\bar{\rho}_{12}}} .
\end{gathered}
$$

Comparing these expressions to (8) provides the first insights into the trade-off between achievable transmission rates and radio resource consumption inherent to coordination. When $\bar{\rho}_{1}, \bar{\rho}_{2}, \bar{\rho}_{12} \rightarrow 0$, we get $\gamma_{i} \rightarrow R_{i}^{\prime}$ in the coordination zone and $\gamma_{i} \rightarrow R_{i}$ elsewhere: there is a throughput gain of $R_{i}^{\prime} / R_{i}$ for class $i$ in the coordination zone, as expected. When load increases, the coordination scheme may be detrimental. In the case for two symmetric sectors for instance, the stability condition (15) is stricter than the usual condition $\bar{\rho}_{k}<1$ in each sector $k$ whenever $\bar{R}_{12}^{\prime}<2 \bar{R}_{12}$, where $\bar{R}_{12}$ denotes the mean transmission rate in the coordination region in the absence of coordination:

$$
\bar{R}_{12}=\frac{\sum_{i \in C_{12}} p_{i}}{\sum_{i \in C_{12}} \frac{p_{i}}{R_{i}}} .
$$




\subsection{Coordinating three sectors}

We now consider the practically interesting case of $K=3$ sectors. The analysis can be readily extended to any number of sectors. As previously, we refer to zone $k, k+1$ as the coordination region between sectors $k$ and $k+1$, for all $k=1,2,3$ (with modulo $K$ notations) and denote by $C_{k, k+1} \subset S_{k} \cap S_{k+1}$ the corresponding classes. Zone $k$ refers to the non-coordination region of sector $k$; it corresponds to classes $S_{k} \backslash\left(C_{k, k-1} \cup C_{k, k+1}\right)$.

Let $\vec{Z}(t)$ be the vector of the number of flows in each zone. When $\vec{Z}(t)=$ $\vec{z}$, the considered scheduler selects UEs in zone 1 a fraction of time:

$$
\phi_{1}(\vec{z})=\frac{z_{1}+z_{23}}{|z|}+\frac{z_{2}}{|z|} \frac{z_{1}+z_{3}}{z_{1}+z_{3}+z_{31}}+\frac{z_{3}}{|z|} \frac{z_{1}+z_{2}}{z_{1}+z_{2}+z_{12}},
$$

and UEs in zone 12 a fraction of time:

$$
\phi_{12}(\vec{z})=\frac{z_{12}}{|z|}+\frac{z_{3}}{|z|} \frac{z_{12}}{z_{1}+z_{2}+z_{12}},
$$

where $|z|$ denotes the total number of users in the whole site. We obtain (19) for instance by observing that a UE in zone 12 is either selected first by the scheduling algorithm, with probability $z_{12} /|z|$, or after the selection of a UE in zone 3 , with probability $z_{3} /|z|$; in the latter case, only UEs in zones 1,2 or 12 can be selected in the same timeslot. Expression (18) can be derived in the same way, and the timeslot allocation in the other zones follow by symmetry.

Since time-sharing is fair in each zone, the system corresponds to a set of six coupled processor-sharing queues with state-dependent service rates (18)(19) and loads defined by (12), as in the case of two sectors. Unfortunately, the service rates violate the balance property so that the system is no longer a Whittle network, see [25]. To get explicit expressions for the flow throughput, we proceed by approximation which we validate numerically. Specifically, we decouple the six zones by neglecting those which are not directly linked by coordination. For instance, we focus the study on zones 1, 2, 12 and neglect the impact of sector 3 , beyond the load induced on sectors 1 and 2. In doing so, we reduce the analysis to the case of two sectors considered above. In particular, the stationary distribution of the number of flows in zones $1,2,12$ is approximated by (14), where the loads of zones 1 and 2 are replaced respectively by $\bar{\rho}_{1}+\bar{\rho}_{31}$ and $\bar{\rho}_{2}+\bar{\rho}_{23}$. We deduce the following approximations for the flow throughputs in zones 1 and 12:

$$
\bar{\gamma}_{1} \approx \bar{R}_{1}\left(1-\bar{\rho}_{1}-\bar{\rho}_{31}-\bar{\rho}_{12}\right),
$$




$$
\bar{\gamma}_{12} \approx \frac{\bar{R}_{12}^{\prime}\left(1-\bar{\rho}_{12}\right)}{1+\frac{\bar{\rho}_{1}+\bar{\rho}_{31}}{1-\bar{\rho}_{1}-\bar{\rho}_{12}-\bar{\rho}_{31}}+\frac{\bar{\rho}_{2}+\bar{\rho}_{23}}{1-\bar{\rho}_{2}-\bar{\rho}_{12}-\bar{\rho}_{23}}}
$$

under the stability condition:

$$
\begin{aligned}
& \bar{\rho}_{1}+\bar{\rho}_{12}+\bar{\rho}_{31}<1, \\
& \bar{\rho}_{2}+\bar{\rho}_{12}+\bar{\rho}_{23}<1, \\
& \bar{\rho}_{3}+\bar{\rho}_{31}+\bar{\rho}_{23}<1 .
\end{aligned}
$$

Importantly, the analysis is consistent in the sense that the expression for the flow throughput in zone 1 is the same whether the approximation is applied to zones $1,2,12$ or to zones $1,3,31$. Per-class flow throughputs follow by replacing mean transmission rates by per-class transmission rates, as in the case of two sectors.

In order to validate the analytical approximation, we consider an isotropic site with three symmetric sectors. The system is then defined by the following three dimensionless parameters:

- $\alpha$, the fraction of traffic in the coordination zone,

- $\beta$, the ratio of mean service rates in the coordination region with and without coordination, referred to as the coordination gain.

- $\eta$, the ratio of the mean service rates experienced in and out of the coordination region, in the reference system without coordination.

We have:

$$
\alpha=\frac{\bar{\lambda}_{12}}{\bar{\lambda}_{1}+\bar{\lambda}_{12}}, \quad \beta=\frac{\bar{R}_{12}^{\prime}}{\bar{R}_{12}}, \quad \eta=\frac{\bar{R}_{12}}{\bar{R}_{1}} .
$$

The normalized flow throughput averaged over the whole sector is shown in figure 2 for $\alpha=0.3, \beta=1.4$ and $\eta=0.5$. The chosen throughput unit is the harmonic mean transmission rate in the absence of coordination.

In view of $(9)$, the flow throughput is then equal to $1-\bar{\rho}$ in the absence of coordination, where $\bar{\rho}$ denotes the common load of each sector. In the presence of coordination, the harmonic mean transmission rate is given by:

$$
\frac{1-\alpha+\frac{\alpha}{\eta}}{1-\alpha+\frac{\alpha}{\beta \eta}} \approx 1.15
$$




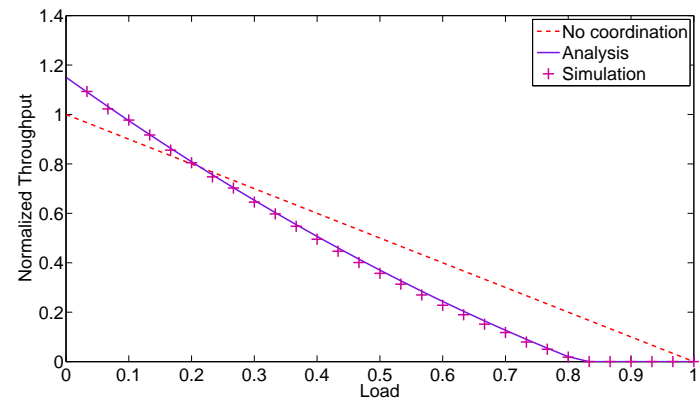

Figure 2: Validation of the approximation (static coordination scheme).

corresponding to an average gain of $15 \%$, while the maximum load is:

$$
\frac{1-\alpha+\frac{\alpha}{\eta}}{1-\alpha+2 \frac{\alpha}{\beta \eta}} \approx 0.83
$$

corresponding to a loss of $17 \%$. The analytical expression derived from (20) and (21) is compared with the simulation results of $10^{6}$ jumps of the Markov process. We observe that the approximation is very accurate.

\subsection{Numerical results}

For the numerical applications, we focus on a HSPA+ system where coordination is performed at sector-level according to the multi-flow transmission.

\subsubsection{Transmission rates}
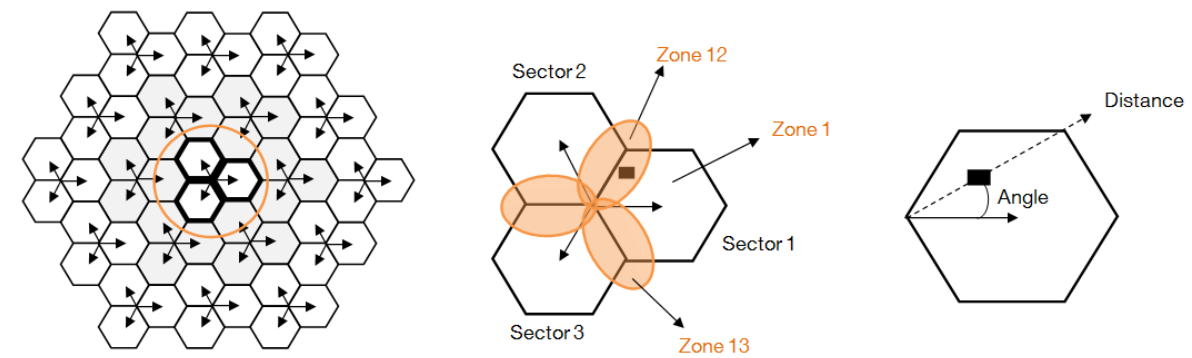

Figure 3: Network model, site model and sector model.

We consider a network of 20 homogeneous tri-sector sites as shown in figure 3. The transmission rates are observed in different positions of some 
reference cell with respect to the distance to the $\mathrm{BS} d$ and the angle relative to the main direction of the antenna $a$. The rates are reported in both cases (with and without coordination) according to the parameters summarized in table 1 and assuming that:

- the rate function $f$, which takes fast fading into account, is that given in $[32]$,

- the type 3i receiver (diversity equalizer with interference awareness [3]) is able to reduce/suppress the interference from the coordinated sectors,

- when the multi-flow transmission is activated, the coordination region is assumed to represent a fraction $\alpha$ of the sector area, as illustrated in figure 3 for a homogeneous network. Note that, in irregular networks, the coordination area does not necessarily have a regular shape in the overlapping area between the two sectors.

Table 1: Simulation parameters

\begin{tabular}{|l|c|}
\hline Parameter & Value \\
\hline site layout & Hexagonal grid, 20 sites, 3 sectors per site \\
\hline Inter-site distance & $500 \mathrm{~m}$ \\
\hline Carrier frequency band & COST-231 Hata \\
\hline Propagation model & PA3 \\
\hline Radio channel & dense urban \\
\hline Morphology & deep indoor \\
\hline Penetration type & $21 \mathrm{~dB}$ \\
\hline Penetration margin & type 3i \\
\hline UE Receiver Type & $0 \mathrm{~dB}$ \\
\hline UE Antenna gain & $43 \mathrm{dBm}$ \\
\hline NodeB maximum power & $18 \mathrm{~dB}$ \\
\hline NodeB Antenna gain & Intra-site \\
\hline Multi-flow operation & $100 \%$ \\
\hline Load at neighboring sites & 12 for $\alpha=30 \%$ \\
\hline Coordination threshold & \\
\hline
\end{tabular}

In order to illustrate the gain of multi-flow on the transmission rates and the impact of the receiver, we plot in figure 4 the achievable values with 
respect to the distance between the user and the cell site with a perfect receiver (corresponding to $\epsilon=0 \%$ ). In figure 5 , we plot the achievable rates for the following cases:

- for an angle of $10^{\circ}$ with the main serving beam, corresponding to positions that are never coordinated.

- for an angle of $60^{\circ}$ with the main serving beam corresponding to positions that are coordinated when MF is activated.

- with a perfect receiver (figure 5a) vs. a receiver that cancels only $50 \%$ of interference (figure 5b).

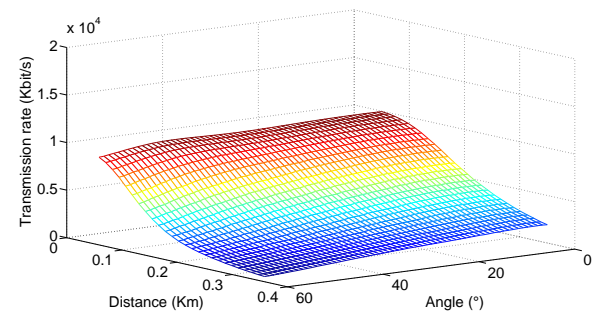

(a) Without coordination.

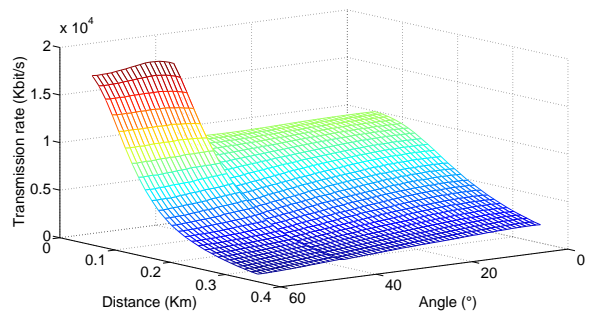

(b) With coordination $(\alpha=30 \%)$.

Figure 4: Achievable rates in the reference cell with a perfect receiver $(\epsilon=0 \%)$.

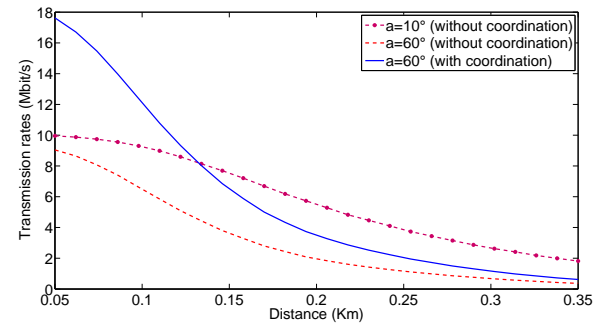

(a) With a perfect receiver $(\epsilon=0 \%)$.

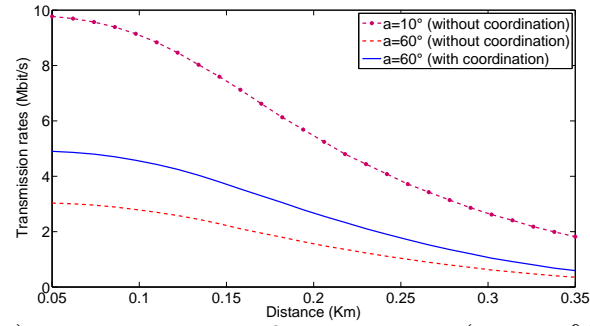

(b) With an imperfect receiver $(\epsilon=50 \%)$.

Figure 5: Transmission rates in different cases.

We see that higher rates are achieved when MF is performed in the coordination area, especially with a perfect receiver. Note that at high distance, 
users at sector boundaries suffer from high interference from neighboring sites in addition to the intra-site interference which makes the transmission rates low even with a perfect receiver.

\subsubsection{Static coordination performance}

Using the transmission rates obtained in $\S 6.4 .1$ at different positions in the reference site and according to (20) and (21), we get the flow throughputs for different traffic conditions, as shown in figure 6 . In this and subsequent figures, the flow throughput is averaged either over the whole sector or over the cell edge, corresponding to $5 \%$ of users with the lowest throughputs. The offered traffic corresponds to the traffic intensity per sector $\bar{\lambda}_{k} \sigma$.

With a perfect 3 i receiver, we observe a significant throughput improvement at low load (up to $25 \%$ at the cell edge, $20 \%$ on average) at the expense of a reduction of the stability region. Static coordination is only beneficial at low load. Figure 6 shows also the throughputs obtained with an imperfect receiver referring to the case where $\epsilon=50 \%$ (i.e. only $50 \%$ of the interference are suppressed).

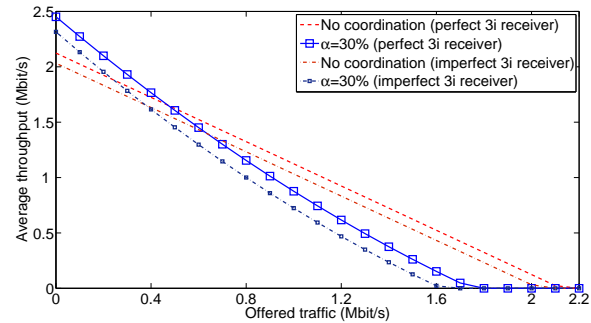

(a) Average flow throughput.

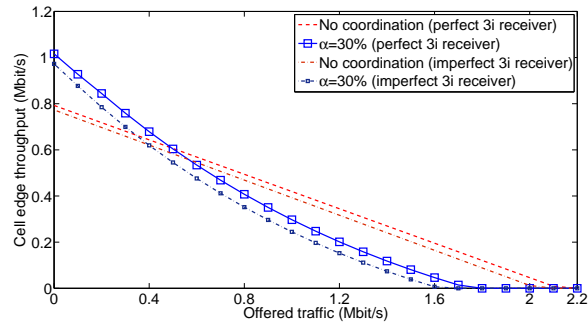

(b) Cell edge flow throughput.

Figure 6: Flow throughput vs. offered traffic $(\alpha=30 \%)$.

We notice that the throughput decreases at the same speed and that the system capacity is slightly decreased by around $7 \%$. Therefore, in the following, we will only consider perfect 3 i receiver for the performance evaluation.

\subsubsection{Sensitivity to the coordination threshold}

We give in figure 7 the average flow throughput for all possible values of $\alpha$ ranging from 0 to 1 in a given site with three sectors. We observe that the coordination region defined by the threshold $\delta$ has a strong impact on performance, depending on the offered traffic. 


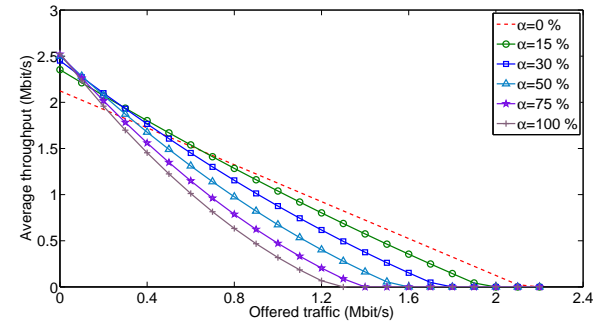

(a) Average flow throughput.

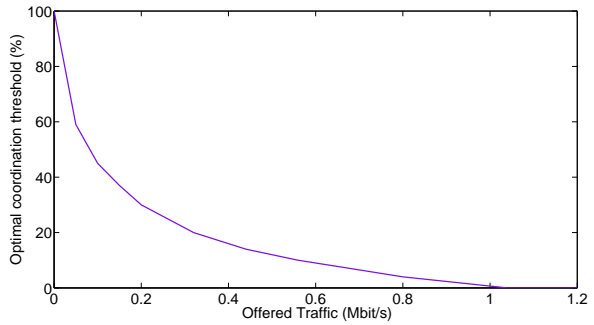

(b) Optimal coordination threshold.

Figure 7: Impact of the coordination threshold on the average flow throughput under the static scheme.

The optimal coordination region is given in figure 7 with respect to the traffic intensity. Observe that no coordination should be performed beyond a traffic intensity of $1 \mathrm{Mbit} / \mathrm{s}$, corresponding to a cell load of 50\%. This indicates that MF is too sensitive to the coordination threshold when coordination is systematically performed in the overlapping zone. This motivates the introduction of a more dynamic, load dependent scheme, as the one discussed in the next section.

\section{Dynamic coordination scheme}

In this section, we investigate a dynamic scheme which performs coordination according to the resource availability in the network. Indeed, a UE in the coordination region of two sectors receives data from the non-serving BS only if the latter has no UE to serve in its serving zone.

\subsection{Scheduling algorithm}

The dynamic scheme consists in allowing a sector to coordinate and serve a UE that is not located in its service area only if it has no other UE to serve. For simplicity, we suppose that when there is a competition between users requiring coordination from the same sector, the choice is done uniformly at random. We shall see that this simple scheme is sufficient to improve performance at any load. The impact of opportunistic scheduling algorithms like Proportional Fair is given in section 8. 


\subsection{Coordinating two sectors}

Like for the static scheme, we start with the case $K=2$. We use the same notation, except that we now differentiate between zone 12, consisting of classes in the coordination region served by sector 1 , and zone 21 , consisting of classes in the coordination region served by sector 2 .

We denote by $Y_{1}(t)=Z_{1}(t)+Z_{12}(t)$ and $Y_{2}(t)=Z_{2}(t)+Z_{21}(t)$ the numbers of flows in sector 1 and 2 at time $t$, respectively. The system is more complex than in the static case since the two processor-sharing queues associated with each sector now interact at the class level. Specifically, the queuing system is described by the following transition rates of the Markov process $\vec{Z}(t)$ between two states $\vec{z}$ and $\vec{z}^{*}$ :

$$
q\left(\vec{z}, \vec{z}^{\prime}\right)= \begin{cases}\lambda_{j} & \text { for } \vec{z}^{\prime}=\vec{z}+\vec{e}_{j} j \in\{1,2,12,21\} \\ \phi_{j}(\vec{z}) \frac{\bar{R}_{j}}{\sigma} & \text { for } \vec{z}^{\prime}=\vec{z}-\vec{e}_{j}, j \in\{1,2\} \\ \phi_{12}(\vec{z}) \frac{\bar{R}_{12}}{\sigma} & \text { for } \vec{z}^{\prime}=\vec{z}-\vec{e}_{12}, y_{2}>0 \\ \phi_{12}(\vec{z}) \frac{\beta R_{12}}{\sigma} & \text { for } \vec{z}^{\prime}=\vec{z}-\vec{e}_{12}, y_{2}=0 \\ \phi_{21}(\vec{z}) \frac{\vec{R}_{21}}{\sigma} & \text { for } \vec{z}^{\prime}=\vec{z}-\vec{e}_{12}, y_{1}>0 \\ \phi_{21}(\vec{z}) \frac{\beta \bar{R}_{21}}{\sigma} & \text { for } \vec{z}^{\prime}=\vec{z}-\vec{e}_{12}, y_{1}=0\end{cases}
$$

with $\phi_{j}(\vec{z})=\frac{z_{j}}{y_{k}}$, for each zone $j$ in sector $k$.

We decouple the system by replacing the state-dependent coordination gain of each class by its mean, so that the coordination gain in zones 12 and 21 are respectively given by:

$$
\begin{aligned}
& \bar{\beta}_{12}=P\left(Y_{2}>0\right)+\frac{\bar{R}_{12}^{\prime}}{\bar{R}_{12}} P\left(Y_{2}=0\right), \\
& \bar{\beta}_{21}=P\left(Y_{1}>0\right)+\frac{\bar{R}_{21}^{\prime}}{\bar{R}_{21}} P\left(Y_{1}=0\right) .
\end{aligned}
$$

Sectors 1 and 2 now behave as two independent multi-class processor-sharing queues with respective loads:

$$
\bar{\rho}_{1}=\frac{\bar{\lambda}_{1} \sigma}{\bar{R}_{1}}+\frac{\bar{\lambda}_{12} \sigma}{\bar{\beta}_{12} \bar{R}_{12}}, \quad \bar{\rho}_{2}=\frac{\bar{\lambda}_{2} \sigma}{\bar{R}_{2}}+\frac{\bar{\lambda}_{21} \sigma}{\bar{\beta}_{21} \bar{R}_{21}} .
$$

In particular, we have:

$$
P\left(Y_{1}>0\right)=\bar{\rho}_{1}, P\left(Y_{2}>0\right)=\bar{\rho}_{2},
$$


from which we deduce $\bar{\beta}_{12}$ and $\bar{\beta}_{21}$.

The flow throughputs in sector 1 are then given by:

$$
\begin{gathered}
\bar{\gamma}_{1}=\bar{R}_{1}\left(1-\bar{\rho}_{1}\right), \\
\bar{\gamma}_{12}=\bar{\beta}_{12} \bar{R}_{12}=\left(\bar{R}_{12} \bar{\rho}_{2}+\bar{R}_{12}^{\prime}\left(1-\bar{\rho}_{2}\right)\right)\left(1-\bar{\rho}_{1}\right),
\end{gathered}
$$

using (9), we deduce the class- $i$ flow throughput as follows:

$$
\begin{gathered}
\forall i \in C_{1}, \quad \gamma_{i}=R_{i}\left(1-\bar{\rho}_{1}\right), \\
\forall i \in C_{12}, \quad \gamma_{i}=\left(R_{i} \bar{\rho}_{2}+R_{i}^{\prime}\left(1-\bar{\rho}_{2}\right)\right)\left(1-\bar{\rho}_{1}\right),
\end{gathered}
$$

using (8). Expressions for sector 2 follow by symmetry.

It is worth observing that the stability region is preserved: since $\bar{\beta}_{12}, \bar{\beta}_{21}>$ 1 , the stability condition $\bar{\rho}_{1}<1, \bar{\rho}_{2}<1$ is not stricter than that in the absence of coordination. Both stability regions in fact coincide since $\bar{\beta}_{12}, \bar{\beta}_{21} \rightarrow 1$ at high load (when $\bar{\rho}_{1}, \bar{\rho}_{2} \rightarrow 1$ ).

\subsection{Coordinating three sectors}

The approach is similar for $K$ sectors. We take $K=3$ to simplify the notation. The coordination gain in zone 12 is given by:

$$
\begin{aligned}
\bar{\beta}_{12} & =P\left(Y_{2}>0\right)+\frac{\bar{R}_{12}^{\prime}}{\bar{R}_{12}} P\left(Y_{2}=0, Y_{3}=0\right) \\
& +\frac{1}{2}\left(1+\frac{\bar{R}_{12}^{\prime}}{\bar{R}_{12}}\right) P\left(Y_{2}=0, Y_{3}>0\right) E\left(\frac{Z_{32}}{Y_{3}} \mid Y_{3}>0\right) \\
& +\frac{\bar{R}_{12}^{\prime}}{\bar{R}_{12}} P\left(Y_{2}=0, Y_{3}>0\right) E\left(\frac{Y_{3}-Z_{32}}{Y_{3}} \mid Y_{3}>0\right),
\end{aligned}
$$

which can be expressed more simply by:

$$
\begin{aligned}
\bar{\beta}_{12}= & P\left(Y_{2}>0\right)+\frac{\bar{R}_{12}^{\prime}}{\bar{R}_{12}} P\left(Y_{2}=0\right) \\
& +\frac{1}{2}\left(1-\frac{\bar{R}_{12}^{\prime}}{\bar{R}_{12}}\right) P\left(Y_{2}=0, Y_{3}>0\right) E\left(\frac{Z_{32}}{Y_{3}} \mid Y_{3}>0\right) .
\end{aligned}
$$

Indeed, a UE in zone 12 benefits from coordination only if sector 2 is empty and no UE in zone 32 benefits from coordination. When sector 2 is empty and UEs in zones 12 and 32 are scheduled, we assume that sector 2 
selects sector 1 or 3 uniformly at random for coordination, hence the factor $\frac{1}{2}$. Sectors 1, 2, 3 behave as three independent multi-class processor-sharing queues with respective loads:

$$
\begin{gathered}
\bar{\rho}_{1}=\frac{\bar{\lambda}_{1} \sigma}{\bar{R}_{1}}+\frac{\bar{\lambda}_{12} \sigma}{\bar{\beta}_{12} \bar{R}_{12}}+\frac{\bar{\lambda}_{13} \sigma}{\bar{\beta}_{13} \bar{R}_{13}}, \\
\bar{\rho}_{2}=\frac{\bar{\lambda}_{2} \sigma}{\bar{R}_{2}}+\frac{\bar{\lambda}_{21} \sigma}{\bar{\beta}_{21} \bar{R}_{21}}+\frac{\bar{\lambda}_{23} \sigma}{\bar{\beta}_{23} \bar{R}_{23}}, \\
\bar{\rho}_{3}=\frac{\bar{\lambda}_{3} \sigma}{\bar{R}_{3}}+\frac{\bar{\lambda}_{31} \sigma}{\bar{\beta}_{31} \bar{R}_{31}}+\frac{\bar{\lambda}_{32} \sigma}{\bar{\beta}_{32} \bar{R}_{32}} .
\end{gathered}
$$

In particular, we have:

$$
\begin{aligned}
& P\left(Y_{2}>0\right)=\bar{\rho}_{2}, P\left(Y_{3}>0\right)=\bar{\rho}_{3}, \\
& E\left(\frac{Z_{32}}{Y_{3}} \mid Y_{3}>0\right)=\frac{\bar{\rho}_{32}}{\bar{\rho}_{3}+\bar{\rho}_{31}+\bar{\rho}_{32}},
\end{aligned}
$$

with:

$$
\bar{\rho}_{32}=\frac{\bar{\lambda}_{32} \sigma}{\bar{\beta}_{32} \bar{R}_{32}}
$$

from which we deduce the coordination gains $\bar{\beta}_{j}$ for each coordination zone $j=12,21,23,32,13,31$. The stability condition $\bar{\rho}_{1}<1, \bar{\rho}_{2}<1, \bar{\rho}_{3}<1$ is the same as without coordination and the flow throughputs in zones 1 and 12 are given by:

$$
\begin{gathered}
\bar{\gamma}_{1}=\bar{R}_{1}\left(1-\bar{\rho}_{1}\right), \\
\bar{\gamma}_{12}=\bar{\beta}_{12} \bar{R}_{12}=\left(\bar{R}_{12} \bar{\rho}_{2}+\bar{R}_{12}^{\prime}\left(1-\bar{\rho}_{2}\right)+\frac{\bar{R}_{12}-\bar{R}_{12}^{\prime}}{2}\left(1-\bar{\rho}_{2}\right) \bar{\rho}_{32}\right)\left(1-\bar{\rho}_{1}\right) .
\end{gathered}
$$

We obtain the class- $i$ flow throughput:

$$
\begin{gathered}
\forall i \in C_{1}, \quad \gamma_{i}=R_{i}\left(1-\bar{\rho}_{1}\right), \\
\forall i \in C_{12}, \quad \gamma_{i}=\left(R_{i} \bar{\rho}_{2}+R_{i}^{\prime}\left(1-\bar{\rho}_{2}\right)+\frac{R_{i}-R_{i}^{\prime}}{2}\left(1-\bar{\rho}_{2}\right) \bar{\rho}_{32}\right)\left(1-\bar{\rho}_{1}\right) .
\end{gathered}
$$


The expressions for the other zones follow by symmetry. In the symmetric case, with the parameters $\alpha, \beta, \eta$ introduced in $\S 6.3$, the common coordination gain $\bar{\beta}$ satisfies the equation:

$$
\bar{\beta}=\bar{\rho}+\beta(1-\bar{\rho})+\frac{1}{4}(1-\beta) \bar{\rho}(1-\bar{\rho}) \frac{\alpha}{\alpha+(1-\alpha) \eta \bar{\beta}},
$$

where $\bar{\rho}$ denotes the common sector load. The stability condition is $\bar{\rho}<1$ and the flow throughputs in zone 1 and zone 12 are respectively given by:

$$
\bar{\gamma}_{1}=\bar{R}_{1}(1-\bar{\rho}) \quad \text { and } \quad \bar{\gamma}_{12}=\bar{\beta} \bar{R}_{12}(1-\bar{\rho}) .
$$

The normalized flow throughput averaged over the whole site is shown in figure 8 for $\alpha=0.3, \beta=1.4$ and $\eta=0.5$. As in Figure 2, the throughput unit is the mean achievable data rate in the absence of coordination. The flow throughput derived from the solution of the fixed-point equation (27) is compared with the simulation results of $10^{6}$ jumps of the Markov process, assuming an exponential flow size distribution. Again, the approximation is very accurate and performance is practically insensitive to the flow size distribution.

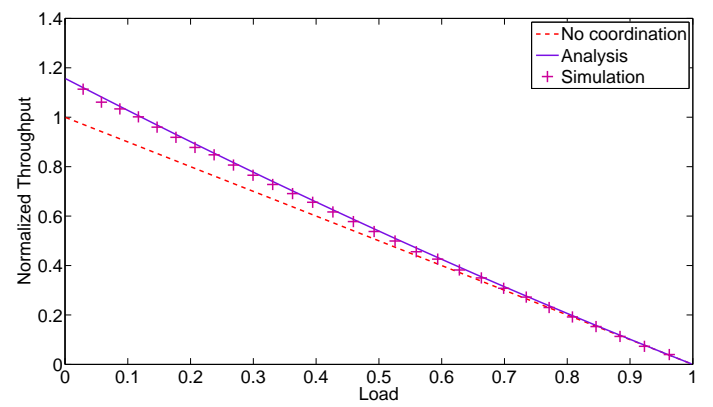

Figure 8: Validation of the approximation (dynamic coordination scheme).

\subsection{Numerical results}

We use the HSPA+ system performance obtained details in 6.4.1 with the analytical study of the dynamic coordination scheme derived previously. 


\subsubsection{Dynamic coordination performance}

Figure 9 shows the flow throughputs averaged over the site and at the cell edge, respectively, using the transmission rates of §6.4.1. The first observation is that, in contrast with the static scheme, the dynamic scheme always improves performance regardless of the load. Indeed, while the dynamic scheme is able to achieve the same gains as the static scheme at low loads (up to $25 \%$ for the cell edge, $20 \%$ on average), it preserves the stability region and prevents throughput degradation at high loads.

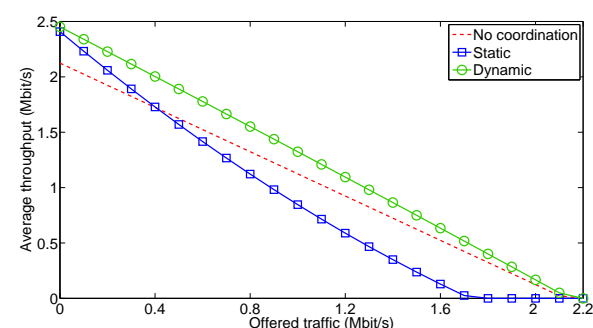

(a) Average throughput.

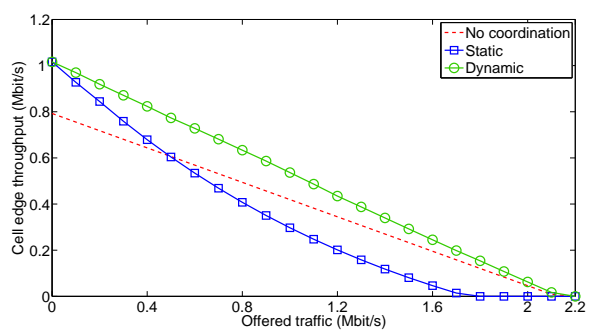

(b) Cell edge throughput.

Figure 9: Flow throughput under the static and dynamic schemes $(\alpha=30 \%)$.

\subsubsection{Sensitivity to coordination threshold}

Figure 10 shows the average throughput achievable with different values of $\alpha$. We see that changing the coordination region area has only a slight impact on the performance of the dynamic scheme since it enables coordination only when resources are available.

This solves a major problem of the static coordination scheme that is its large sensitivity to the coordination threshold as was illustrated in section §6.4.3. This also eases the system engineering as it would be sufficient to set the coordination threshold to a fixed value, e.g. corresponding to the handover region, without having to adapt it to the traffic load.

\subsubsection{Coordinating non-uniformly loaded sectors}

While we considered up to this point the case of uniform traffic load across the site, we now move to the case of non-uniformly loaded sectors. Indeed, one of the major advantages of cell coordination is that it allows to naturally balance the load between neighboring cells by allowing a low loaded sector to handle a part of the traffic of its highly loaded neighbors. 


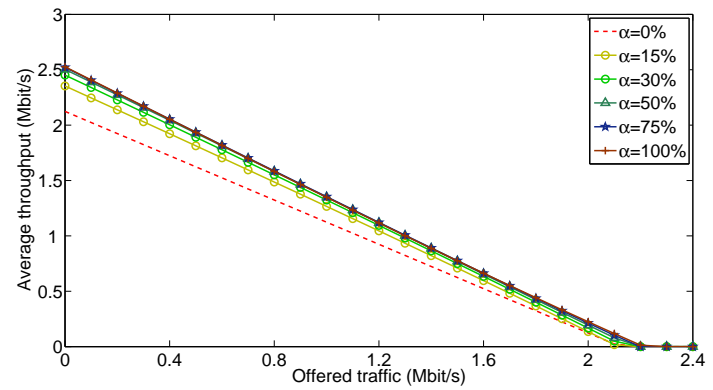

Figure 10: Impact of the coordination threshold on the average flow throughput under the dynamic scheme.

Note that the dynamic coordination scheme tends particularly to balance load across sectors as it benefits from the idle periods of time in order to help its neighbors in handling their traffic.

Figure 11 shows this ability of multi-flow transmission to balance load across non-uniformly loaded sectors. It compares the case of uniformly loaded sectors (figure 9) to the case where one sector of the site is highly loaded and the two others have a traffic that is $80 \%$ lower. Results show that the average throughput is improved and the system stability is enlarged when neighboring sectors are relatively less loaded.

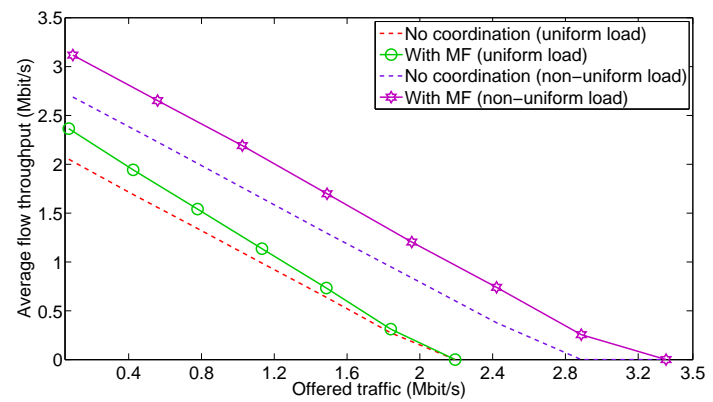

Figure 11: Average flow throughput under dynamic scheme in different load conditions.

Note that the performance of the baseline system (without coordination) is different in the two cases as in the case of non-uniform traffic, the inter-cell interference is lower, leading to better baseline performance. 


\subsubsection{Coordinating in a homogeneous network}

So far, we have considered a constant transmission rate referring to the case where neighboring sites are fully loaded (see table 1). We suppose now that the transmission rate depends on the load at neighboring sites, through interference.

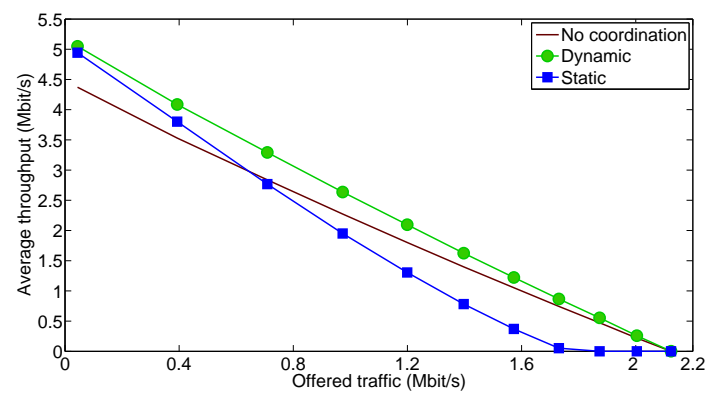

Figure 12: Average flow throughput in a network with homogeneous load.

We show in figure 12 the performance in terms of average throughput with static and dynamic coordination. The 11 points correspond to the increasing values of the load from 0.01 to 1 . We notice the same performance of coordination over the classical system without coordination. However, we see that the throughput achieves higher values at low loads due to less interference from neighboring sites.

\section{Impact of opportunistic scheduling}

Finally, we study the impact of opportunistic scheduling, namely proportional fair, on the coordination gains.

\subsection{Proportional fair scheduler}

We recall in this section the principles of the Proportional Fair (PF) scheduler [28]. The scheduling decision is based on the instantaneous throughput $r_{u}(t)$ of user $u$ at time $t$ and on the average throughput $R_{u}(t)$ of user $u$ at time $t$, evaluated through the moving average:

$$
R_{u}(t)=(1-\theta) R_{u}(t-1)+\theta r_{u}(t),
$$

for some fixed time parameter $\theta$. 
The PF policy schedules the user with the best relative radio conditions, that is with the maximum ratio:

$$
\frac{r_{u}(t)}{R_{u}(t)}
$$

The scheduling gain over a blind algorithm like round-robin depends on the number of users $n$ and on the channel model.

In the following, we consider Rayleigh fast fading and we assume that the rate variations around the mean are symmetric in all classes [33]. so that the gain $G(n)$ is approximately equal to the $\min \left(3,1+\ldots+\frac{1}{n}\right)$ for $n$ users [34]. We limit this gain to 3 which corresponds to $n=10$ users per sector. Note that the gain can be obtained numerically for more realistic channels like PA3 or VA3 channels [35].

For the classical case without coordination, a user scheduled in sector $k$ with $\bar{x}_{k}$ active flows has the best relative radio conditions among all users of the same sector, corresponding to a scheduling gain of $G\left(\bar{x}_{k}\right)$. The stationary distribution of the number of flows in the site can be expressed by:

$$
\hat{\pi}(\vec{x})=\prod_{k=1}^{K}\left(1-\bar{\rho}_{k}\right) \frac{\bar{x}_{k} !}{\prod_{i \in S_{k}} x_{i} !} \frac{1}{\prod_{n=1}^{\bar{x}_{k}} G(n)} \prod_{i \in S_{k}} \rho_{i}^{x_{i}} .
$$

\subsection{Static coordination scheme}

We start with the simple case of two sectors. Let $|z|=z_{1}+z_{2}+z_{12}$ denotes the total number of users in the site. Considering proportional fair algorithm, a user in zone 1 can be scheduled first, or after the selection of a user in zone 2. Accordingly, it has the best relative radio conditions among either all users of the site or only $z_{1}$ users, corresponding to a scheduling gain of $G(|z|)$ with $|z|=z_{1}+z_{2}+z_{12}$ or $G\left(z_{1}\right)$, respectively. However, a user scheduled in zone 12 has necessarily the best relative radio conditions among all users, corresponding to the scheduling gain of $G(|z|)$. In view of (10)-(11), users in zone 1 and zone 12 are selected with a normalized service rates of:

$$
\hat{\phi}_{1}(\vec{z})=\frac{z_{1}}{z_{1}+z_{2}+z_{12}} G(|z|)+\frac{z_{2}}{z_{1}+z_{2}+z_{12}} G\left(z_{1}\right)
$$

and

$$
\hat{\phi}_{12}(\vec{z})=\phi_{12}(\vec{z}) G(|z|) .
$$

In this case, we notice that the service rates do not satisfy the balance property and the corresponding Markov process is no longer reversible. 
This approach can be extended to any number of sectors and particularly to $K=3$ sectors. In view of (18)-(19), UEs in zone 1 and zone 12 are selected with a normalized service rates of:

$$
\begin{gathered}
\hat{\phi}_{1}(\vec{z})=\frac{z_{1}}{|z|} G(|z|)+\frac{z_{23}}{|z|} G\left(z_{1}\right)+\frac{z_{2}}{|z|}\left(\frac{z_{1} G\left(z_{1}+z_{3}+z_{31}\right)}{z_{1}+z_{3}+z_{31}}+\frac{z_{3} G\left(z_{1}\right)}{z_{1}+z_{3}+z_{31}}\right) \\
+\frac{z_{3}}{|z|}\left(\frac{z_{1} G\left(z_{1}+z_{2}+z_{12}\right)}{z_{1}+z_{2}+z_{12}}+\frac{z_{2} G\left(z_{1}\right)}{z_{1}+z_{2}+z_{12}}\right), \\
\hat{\phi}_{12}(\vec{z})=\frac{z_{12}}{|z|} G(|z|)+\frac{z_{3}}{|z|} \frac{z_{12} G\left(z_{1}+z_{2}+z_{12}\right)}{z_{1}+z_{2}+z_{12}},
\end{gathered}
$$

and so on for the selected user in other zones.

We proceed by simulation of the Markov process. The corresponding normalized flow throughput is shown in figure 13 for $\alpha=0.3, \beta=1.4$ and $\eta=0.5$. We notice an improvement in throughput at low load at the expense of a decrease in the network stability of around $17 \%$ compared to the case without coordination.

\subsection{Dynamic coordination scheme}

Considering proportional fair algorithm, a scheduled user in sector $k$ has the best relative radio conditions in this sector, yielding a scheduling gain equal to $G(n)$ for $n$ users in sector $k$. Using the decoupling approach of $\S 7.3$, we get the stationary distribution of the number of users in sector $k$ :

$$
\pi_{k}\left(y_{k}\right)=\pi_{k}(0) \frac{\bar{\rho}_{k}^{y_{k}}}{\prod_{n=1}^{y_{k}} G(n)},
$$

where $\bar{\rho}_{k}$ denotes the load per sector $k$. We deduce:

$$
P\left(Y_{k}>0\right)=1-\pi_{k}(0)=1-\left(\sum_{y_{k}=0}^{\infty} \frac{\bar{\rho}_{k}^{y_{k}}}{\prod_{n=1}^{y_{k}} G(n)}\right)^{-1},
$$

from which we can derive the average coordination gains using (24), then we can obtain the flow throughputs.

Figure 13 compares the performance of the static scheme, the dynamic scheme and the classical case without coordination under opportunistic scheduling. The conclusions drawn in the case of random scheduling remain valid: The static scheme introduces a decrease in the network stability at high load while the dynamic scheme improves performance at low load while preserving the network stability. Besides, we note that the scheduling gain dominates the coordination gain at high loads. 


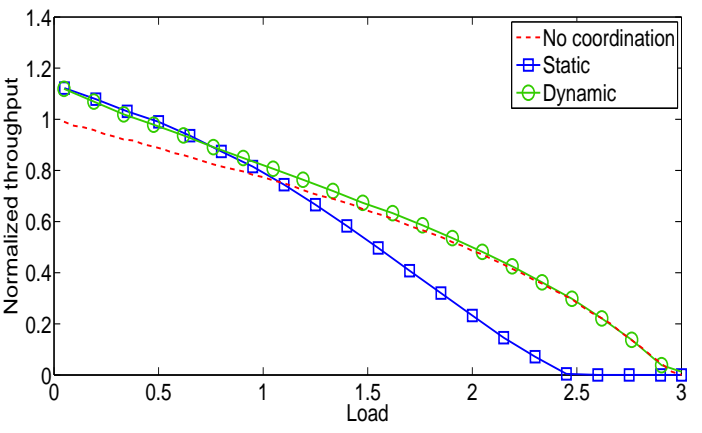

Figure 13: Performance of the static and dynamic schemes under opportunistic scheduling.

\section{Conclusion}

We developed performance evaluation models for several intra-site coordination schemes in HSPA+ networks. We notably showed that using a static scheme, where coordination is systematically performed for users that are in the overlapping areas between the sectors, regardless of the system load, improves throughput at low load at the expense of a reduced stability region when sectors are equally loaded. Besides, we showed that performance is too sensitive to the coordination threshold, i.e. to the size of the coordination area. Alternatively, we investigated a dynamic scheme that takes into account the instantaneous load when making coordination decisions. Our results showed that this scheme improves considerably the throughput performance while preserving the system stability. This dynamic scheme has also shown to be less sensitive to the setting of the coordination threshold, thus easing the system design and management. In addition, we showed that multi-flow transmission is able to balance load between sectors in non homogeneous settings, thus increasing the overall network capacity. We finally showed that these results remain valid for opportunistic scheduling like proportional fair.

Although the focus of this paper was on intra-site coordination applied to macro cells, the framework developed in this work could be used in heterogeneous networks (HetNets) with Remote Radio Heads (RRHs) where centralized coordination schemes can be performed under ideal backhaul assumption. Moreover, this framework could be extended for other systems like LTE-Advanced where Coordinated Multi-point (called CoMP) is used among other techniques (e.g. Multi-User MIMO for instance) in order to 
mitigate intra-site and inter-site interference. This is left as a future work.

\section{References}

[1] T. Bonald, A. Proutiere, Wireless downlink data channels: user performance and cell dimensioning, in: Proceedings of the 9th annual international conference on Mobile computing and networking (MobiCom), ACM, New York, NY, USA, 2003, pp. 339-352.

[2] M. Karakayali, G. Foschini, R. Valenzuela, Network coordination for spectrally efficient communications in cellular systems, Wireless Communications, IEEE 13 (4) (2006) 56 -61.

[3] 3GPP TR 25.872: technical specification group radio access network; high speed packet access (HSDPA) multipoint transmission (Dec. 2011).

[4] 3GPP TR 36.819: Technical specification group radio access network; coordinated multi-point operation for LTE physical layer aspects (release 11) (Dec. 2011).

[5] D. Lee, H. Seo, B. Clerckx, E. Hardouin, D. Mazzarese, S. Nagata, K. Sayana, Coordinated multipoint transmission and reception in LTEadvanced: deployment scenarios and operational challenges, IEEE Communications Magazine 50 (2) (2012) 148-155.

[6] R. Irmer, H. Droste, P. Marsch, M. Grieger, G. Fettweis, S. Brueck, H. P. Mayer, L. Thiele, V. Jungnickel, Coordinated multipoint: Concepts, performance, and field trial results, IEEE Communications Magazine 49 (2) (2011) 102-111. doi:10.1109/MCOM.2011.5706317.

[7] Y. Bejerano, S.-J. Han, Cell breathing techniques for load balancing in wireless LANs, Mobile Computing, IEEE Transactions on 8 (6) (2009) 735-749.

[8] N. Binucci, K. Hiltunen, M. Caselli, Soft handover gain in WCDMA, in: Vehicular Technology Conference, 2000. IEEE-VTS Fall VTC 2000. 52nd, Vol. 3, 2000, pp. 1467-1472 vol.3.

[9] HSPA+ Advanced Smart Networks: Multipoint Transmission, Tech. rep., Qualcomm Incorporated (2011). 
[10] Performance and implementation of SF-DC aggregation, Tech. rep., Qualcomm Incorporated (2011).

[11] The evolution of HSPA: The 3GPP standards progress for fast mobile broadband using HSPA+, Tech. rep., 4gamericas (2011).

[12] D1.8 intermediate report on CoMP (Coordinated Multi-Point) and relaying in the framework of CoMP, Tech. rep., CELTIC, CP5-026 WINNER+ (Oct. 2009).

[13] 3GPP TR 36.814 evolved universal terrestrial radio access (E-UTRA); further advancements for E-UTRA physical layer aspects (3GPP release9) (Mar. 2010).

[14] V. Hytonen, O. Puchko, T. Hohne, T. Chapman, Introduction of multiflow for HSDPA, in: 5th International Conference on New Technologies, Mobility and Security (NTMS), 2012, pp. 1-5. doi:10.1109/NTMS.2012.6208684.

[15] C. Zhang, Y. Chang, S. Liu, D. Yang, System-level analysis and evaluation of SF-DC transmit mode in HSPA system, in: IEEE Wireless Communications and Networking Conference (WCNC), 2012, pp. 1185 -1190. doi:10.1109/WCNC.2012.6213956.

[16] G. Fayolle, R. Iasnogorodski, Two coupled processors: The reduction to a Riemann-Hilbert problem, Probability Theory and Related Fields 47 (1979) 325-351.

[17] T. Bonald, S. Borst, N. Hegde, A. Proutiere, Wireless data performance in multi-cell scenarios, in: Proceedings of ACM SIGMETRICS, 2004, pp. $378-387$.

[18] A. Fehske, G. Fettweis, Aggregation of variables in load models for interference-coupled cellular data networks, in: 2012 IEEE International Conference on Communications (ICC), 2012, pp. 5102-5107.

[19] M. Verloop, R. N. Queija, Asymptotically optimal parallel resource assignment with interference., Queueing Syst. 65 (1) (2010) 43-92. 
[20] P. Osti, P. Lassila, S. Aalto, Optimal intercell coordination for multiple user classes with elastic traffic, in: Next Generation Internet (NGI), 2012 8th EURO-NGI Conference on, 2012, pp. 25-32. doi:10.1109/NGI.2012.6252161.

[21] P. Osti, S. Aalto, P. Lassila, Flow-level modeling and optimization of intercell coordination with dynamic TDD, in: Proceedings of the 10th ACM Symposium on QoS and Security for Wireless and Mobile Networks, Q2SWinet '14, ACM, New York, NY, USA, 2014, pp. 109-118.

[22] A. Vergne, S. Elayoubi, Evaluating the capacity gains from coordinated multipoint transmission and reception, in: IEEE Modeling and Optimization in Mobile, Ad Hoc and Wireless Networks (WiOpt), 2010, pp. 603-609.

[23] A. Khlass, T. Bonald, S. E. Elayoubi, Flow-Level performance of intrasite coordination in cellular networks, in: IEEE Modeling and Optimization in Mobile, Ad Hoc and Wireless Networks (WiOpt), 2013, pp. 303-310.

[24] 3GPP Contribution R1-110563: Simulation framework for system evaluation of muti-point HSDPA (2011).

[25] T. Bonald, A. Proutiere, A queueing analysis of data networks, in: R. J. Boucherie, N. M. van Dijk (Eds.), Queueing Networks: A Fundamental Approach, Vol. 154 of International Series in Operations Research \& Management Science, Springer Verlag, 2011, pp. 643-699.

[26] L. Kleinrock, Queueing Systems, Vol. II: Computer Applications, Wiley Interscience, 1976, (Published in Russian, 1979. Published in Japanese, 1979.).

[27] T. Bonald, M. Feuillet, Network Performance Analysis, ISTE/Wiley, 2011.

[28] H. Kushner, P. Whiting, Convergence of proportional-fair sharing algorithms under general conditions, IEEE Transactions on Wireless Communications 3 (4) (2004) 1250 - 1259. doi:10.1109/TWC.2004.830826.

[29] I. M. Verloop, U. Ayesta, S. C. Borst, Comparison of bandwidth-sharing policies in a linear network, in: Proceedings of the 3rd International 
Conference on Performance Evaluation Methodologies and Tools, ValueTools '08, ICST (Institute for Computer Sciences, Social-Informatics and Telecommunications Engineering), ICST, Brussels, Belgium, Belgium, 2008, pp. 34:1-34:10.

[30] F. P. Kelly, Reversibility and stochastic networks, Wiley series in probability and mathematical statistics, J. Wiley and sons, 1979.

[31] T. Bonald, A. Proutiere, Insensitivity in processor-sharing networks, Perform. Eval. 49 (1/4) (2002) 193-209.

[32] A. Saadani, J.-B. Landre, Realistic performance of HSDPA evolution 64QAM in macro-cell environment, in: Vehicular Technology Conference, 2009. VTC, 2009, pp. 1-5.

[33] S. C. Borst, User-level performance of channel-aware scheduling algorithms in wireless data networks., in: INFOCOM, 2003.

[34] F. Berggren, R. Jantti, A symptotically fair transmission scheduling over fading channel, IEEE Transactions on Wireless Communications 3 (1) (2004) $326-336$.

[35] R. Combes, S.-E. Elayoubi, Z. Altman, Cross-layer analysis of scheduling gains: Application to LMMSE receivers in frequency-selective rayleighfading channels, in: IEEE Modeling and Optimization in Mobile, Ad Hoc and Wireless Networks (WiOpt), 2011, pp. 133-139. 